Proceedings

\title{
Using Agent-Based Modeling to Understand the Emergence and Reproduction of Social Inequalities in Health ${ }^{\dagger}$
}

\author{
Javier Alvarez-Galvez ${ }^{1,2, *}$ and Victor Suarez-Lledo ${ }^{1,3}$ \\ 1 Department of Biomedicine, Biotechnology, and Public Health, University of Cadiz, 11009 Cadiz, Spain; \\ victor.sanz@uca.es \\ 2 University Institute of Research and Innovation in Biomedical Sciences of Cadiz (INiBICA), \\ 11009 Cadiz, Spain \\ 3 University Institute of Research in Social Sustainable Development (INDESS), University of Cadiz, \\ 11406 Cadiz, Spain \\ * Correspondence: javier.alvarezgalvez@uca.es; Tel.: +34-956-019-080 \\ + Presented at the 2nd International Electronic Conference on Environmental Health Sciences, \\ 4-29 November 2019; Available online: https://iecehs-2.sciforum.net/.
}

Published: 1 November 2019

\begin{abstract}
Studies on social inequalities in health present contradictory findings when they attempt to describe and identify the complex societal mechanisms that give rise to poor health outcomes and health inequalities. This work aims to study the mechanism of reproduction of health inequalities among different population groups using agent-based modeling. We combine evidence-based knowledge and survey data to set the simulation model. Our initial findings show that the combination of the most adverse contextual conditions (i.e., negative environmental exposure and the absence of health-care provision) combined with extreme social inequalities in health might increase mortality drastically. The model suggests that, although poor health outcomes may emerge through the action of individual determinants, social inequalities generally emerge and reproduce through non-linear associations and complex multivariate data structures.
\end{abstract}

Keywords: social inequalities; socio-economic status; health inequalities; agent-based models; emergence; complex systems

\section{Introduction}

Despite the present acknowledgment by the scientific community on the relevance of social determinants of health $(\mathrm{SDoH})$ to explain health inequalities, there is persistent uncertainty to explain how contextual and individual level factors are interrelated [1]. Studies have revealed that the higher the socio-economic status (SES), the lower the prevalence of health problems, illness, disease and death [2-4]. However, current works present contradictory findings when they attempt to describe and identify the complex societal mechanisms that give rise to poor health outcomes and health inequalities [5]. A wide attention has been dedicated to analyzing the direct effect of SES indicators on health (e.g., education, income, occupational status, neighborhood, etc.), but the indirect effects with other determinants of health are not commonly described nor totally captured. Determinants of health, such as genetic or environmental ones, are rarely considered as interdependent elements at different levels within a connected whole [6]. Due to the partial study of factors and mechanisms that are dynamic, multidimensional and context-dependent, the association between the SDoH and health inequalities remains empirically inconclusive and theoretically puzzling [7]. 
The fundamental problem of evaluating the effect of environmental and social factors on human health is how to trace the (direct/indirect) causative path and the hidden mechanisms that might describe the process of influence between ecosystems and population health [6]. Research evidence in social and life sciences faces the same analytical problems. The general research approach is too simple to account for the complex interplay between environmental and human systems [1]. Studies on social-environmental determinants of health have mainly been generated by research techniques and methods that were developed to answer specific questions about concrete cause-effect relationships (e.g., to study the impact of education or air pollution on cardiovascular health). Existing empirical evidence commonly assumes a certain kind of bivariate directionality between single predictors (e.g., specific behaviors, environmental exposures, etc.) and specific health outcomes that, theoretically, could be separately explained and understood. Therefore, from this analytical point of view, we cannot describe how a wide set of interdependent social and environmental determinants are connected and related with health outcomes and health inequalities in a single whole [7].

Considering the complex nature of $\mathrm{SDoH}$ and the impossibility of understanding the emergence and reproduction of social inequalities in health from the independent study of single components, recent studies have recognized the need for a complex systems approach to provide new evidence in public health $[1,8]$. This work aims to study the mechanism of reproduction of health inequalities among different population groups using agent-based modeling.

\section{Material and Methods}

\subsection{Data}

In this study, we combined evidence-based knowledge and survey data, from the health inequalities module of the European Social Survey (ESS, 2014) [9], to set an agent-based model (ABM) in Netlogo 6.0.4 (Northwerstern University, Evanston, IL, USA) that aims to study the mechanism of reproduction of health inequalities among different population groups under different simulated scenarios. An ABM is an object-oriented computational model aimed at simulating the behavior of autonomous agents and also their interactions under specific contextual conditions. Like in objectoriented programing, we had to define the properties of some objects (i.e., agents or simulated individuals) and their methods or behaviors (i.e., a specific set of rules that defines their interactions). Inside the simulation model, agents are self-directed individual entities, though they can also represent collective or clustered units, like organizations such as hospitals, schools, or, for example, companies, and even aggregations or groups of individuals such as health-care units in a hospital or departments in a particular company [10-14].

In our ABM, according to scientific literature and evidence-based health data, four basic determinants of health were considered (Table 1): (a) genetic and biological factors; (b) environmental factors; (c) medical care factors; and (d) social circumstances and behavioral factors (i.e., SDoH).

Table 1. Determinants of health.

\begin{tabular}{cccc}
\hline ID & Variables & Measure & Effect Size \\
\hline 1 & Genetic/biological & Initial health & $22 \%$ \\
2 & Environmental & Exposure & $7 \%$ \\
3 & Health care & Accessibility & $11 \%$ \\
4 & Social/behavioral & Survey data & $60 \%$ \\
\hline
\end{tabular}

\subsection{ABM Scenarios}

The effect size for environmental and genetic determinants of health was derived from health science literature, and the relative weight of SDoH indicators was established through the use of survey data and machine learning algorithms. Instead of focusing on the traditional cause-effect approach, we used neural networks (https://cran.rproject.org and http://rstudio.com) to set the 
explanatory power of SDoH $(\mathrm{R} 2=0.22)$, but also to study a new theoretical hypothesis across the implementation of diverse scenarios where different population groups may dynamically interact and affect each other.

\subsection{Model Specification}

In the model, we had two health areas (environment A/environment B) to simulate environmental effects on health. The simulation introduced two types of agents (population group A/population group B) randomly distributed on the map, so that the level of population and gender/ethnic differences might also be defined. All agents had a predefined level of health (0-100), and the same probability to reproduce, get sick and recover (Figure 1). Finally, the effects of health services availability on agents A and B could be defined separately to reflect disparities in health-care provision. Health services were characterized as white-colored agents that were randomly distributed at the beginning of the simulation. These specific agents provided health in a predefined radius to reflect territorial disparities in the access to health facilities.
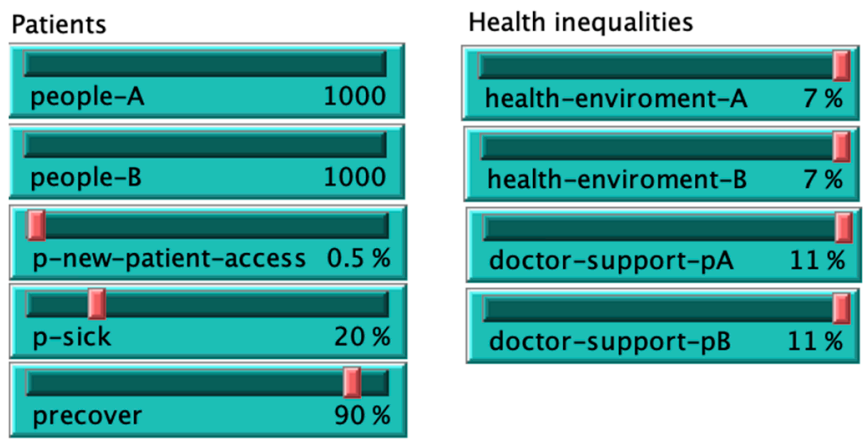

Figure 1. Patients and model initialization panel.

SDoH were collated from the ESS data: family net income, smoking frequency, alcohol frequency, unemployment, discrimination, social capital, childhood problems, education level, fresh diet, physical activity, unmet needs, no. of people living in the house, alternative treatments, accommodation problems and occupational hazards (Figure 2).

Social determinants

\begin{tabular}{|c|c|c|c|c|c|}
\hline physact & 0.0 & alttreat & 0.0 & alcfreq & 0.0 \\
\hline soccap & 0.0 & famincpr & 0.0 & income & 0.0 \\
\hline unmet & 0.0 & praccom & 0.0 & occhaz & 0.00 \\
\hline phouse & 0.0 & isced & 0.0 & etnmin & 0.00 \\
\hline freshdiet & 0.0 & unempl & 0.0 & cigday & 0.000 \\
\hline
\end{tabular}

Figure 2. Social determinants of health panel.

\section{Results}

\subsection{Sick and Death Rates}

Differences in mortality were represented in two basic environments (green environment A/orange environment B). Specifically, it can be observed how dead agents (i.e., black-colored) and sick agents (i.e., red-colored) were more prevalent in environment A (green), which presented poor simulated environmental conditions and a lower proportion of health services. The evolution of population (population A (males) vs. population B (females)), number of sick and dead agents and 
their respective rates were dynamically represented in the ABM model through the following panels (Figure 3):

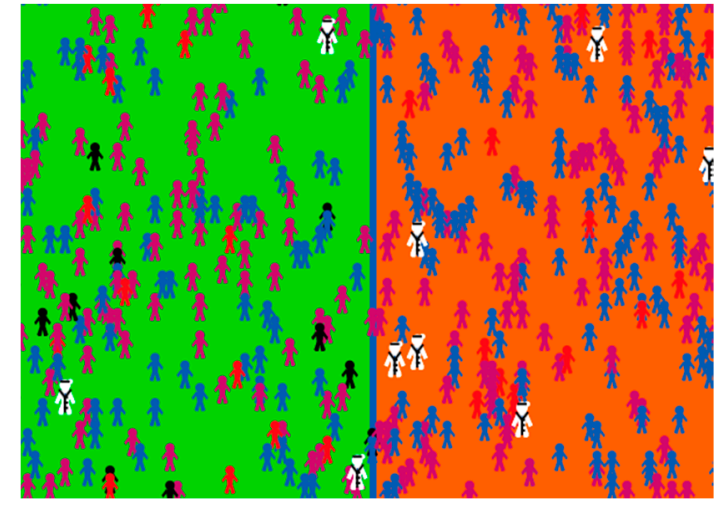

(a)

\begin{tabular}{|c|c|c|c|c|}
\hline \multicolumn{5}{|c|}{ Green environment (A) } \\
\hline People & Sick & Sick Rate & Death & Death Rate \\
\hline 1339 & 142 & 10.6 & 53 & 3.8 \\
\hline \multicolumn{5}{|c|}{ Orange environment (B) } \\
\hline People & Sick & Sick Rate & Death & Death Rate \\
\hline 1445 & 86 & 6 & 9 & 0.6 \\
\hline \multicolumn{5}{|c|}{ Patients A (Males) } \\
\hline People & Sick & Sick Rate & Death & Death Rate \\
\hline 1416 & 111 & 7.8 & 29 & 2 \\
\hline \multicolumn{5}{|c|}{ Patients B (Females) } \\
\hline People & Sick & Sick Rate & Death & Death Rate \\
\hline 1378 & 118 & 8.6 & 34 & 2.4 \\
\hline
\end{tabular}

(b)

Figure 3. Model visualization: (a) sick and dead agents in two simulated environments; (b) population, sick and death rates in environments $\mathrm{A}$ and $\mathrm{B}$, and in two different population groups (males/females).

As we can observe, the model calculated the sick and mortality rates for every agent class and for every environment. In this particular simulation result focused on environmental effects, we can observe that predefining a negative environmental effect of $7 \%$ in the green environment significantly increased differences in morbidity (sick rate 10.6 per 1000 inhabitants in environment A; death rate 6.0 per 1000 inhabitants in environment B) and mortality (sick rate 3.8 per 1000 inhabitants in environment A; death rate 0.6 per 1000 inhabitants in environment B).

\subsection{Mortality Patterns in Four Simulated Scenarios}

Figure 4 presents the results in four basic scenarios: S1 was the baseline model (no effects); S2 simulated environmental differences (i.e., green environment reduced agents' health up to a maximum value of 7\%); S3 added differences in health-care provision (i.e., doctor-agents provided additional health to population A up to a maximum of $11 \%$ ); and $\mathrm{S} 4$ added social inequalities (i.e., the effect of social determinants was set to 0 , so that agents' health was reduced by $22 \%$ ).

As we can observe in Figure 4, mortality differences between population groups A/B and environments $\mathrm{A} / \mathrm{B}$ were low in scenario 1 and the simulated life expectancy of the population (i.e., agents) was about 100 years old. Scenario 2 added environmental differences between environments $\mathrm{A} / \mathrm{B}(7 \%)$ that considerably reduced life expectancy by 25 years in the population living in green environment A. Scenario 3 included differences in the provision of health care (i.e., patients in A received less health), so that inequalities in mortality between populations A and B progressively grew. Finally, scenario 4 considered the effect of SDoH data collated from the ESS: family net income, smoking frequency, alcohol frequency, unemployment, discrimination, social capital, childhood problems, education level, fresh diet, physical activity, unmet needs, no. of people living in the house, alternative treatments, accommodation problems and occupational hazards. In this initial model, we studied the global explanatory effect of SDoH with a maximum explanatory power of $22 \%$ over the final population health of agents in the models. In particular, considering a reduction of these predictors to their joint minimum value and with the objective to simulate the most negative socioeconomic conditions, we can observe that life expectancy decreased under the limit of 50 years on average. 


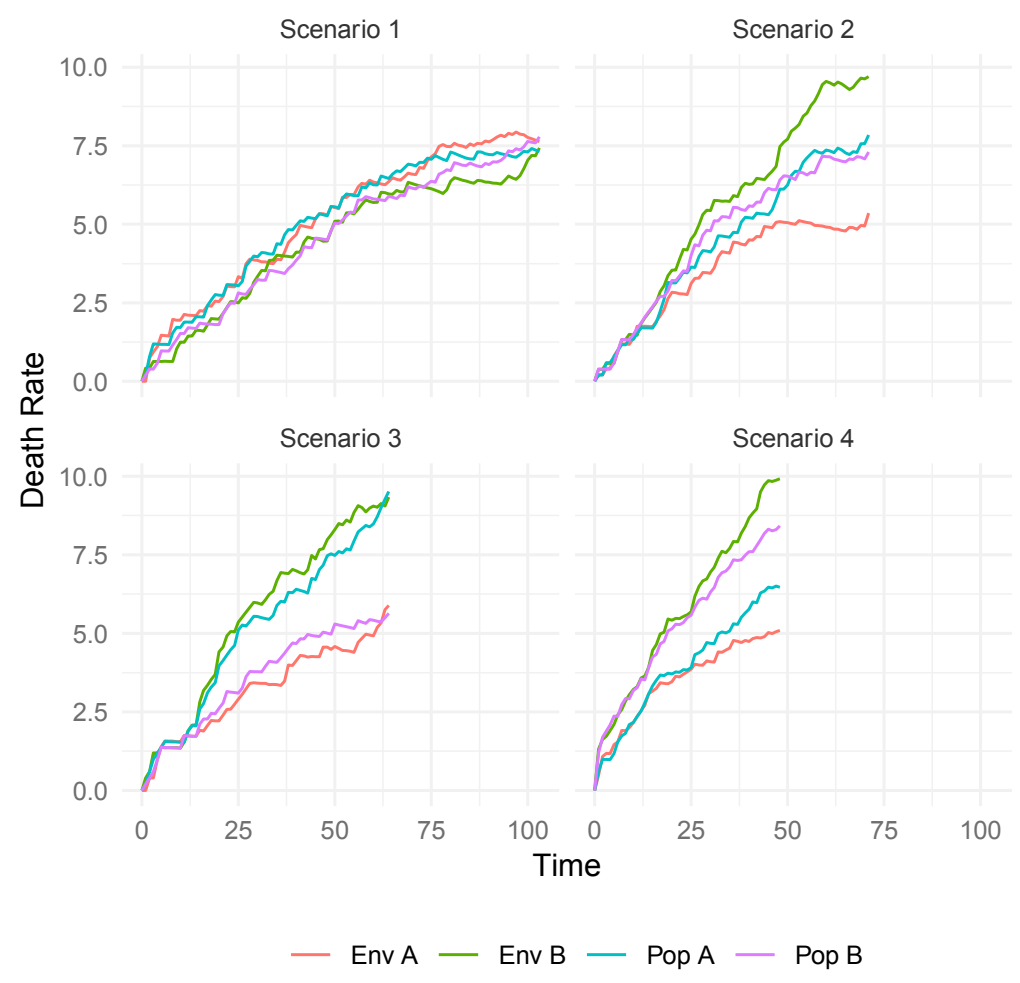

Figure 4. Evolution of death rates in four scenarios.

\section{Discussion}

Although additional models are needed to capture the mechanism of interactions between structural and intermediary determinants of health, our initial findings showed that the combination of the most adverse contextual conditions (i.e., poor environmental circumstances and low healthcare provision) and social inequalities in health might increase mortality drastically (i.e., life expectancy decreased 50 years on average). Therefore, considering that we used a conservative model grounded in evidence-based knowledge and survey data from European societies, it is plausible that health disparities could be even higher in societies where social and economic conditions are more unfavorable.

The present study showed ABM as a suitable tool for dynamically studying health inequalities in ecosystems. These models can identify the mechanism and high-order interactions between multiple interfering factors that are hardly observable using traditional epidemiological approaches. In addition, $\mathrm{ABM}$ may provide a bridge between qualitative and quantitative methods in health and environmental sciences $[1,6,15]$. These models are able to integrate the benefits from different theoretical and methodological approaches, and particular tools such as ABM can give response to the micro-processes that are linked with macro-level dynamics in ecosystems. Using ABM, we can describe processes that are not dynamically observable through isolated methodologies [14]. Thus, the usage of simulated entities (i.e., agents) is fundamental to change our focus from variables/individuals to a new system thinking approach in health and environmental sciences, but also to understand the underlying mechanism of social ecosystems such as self-organization patterns, emergent social phenomena, processes of human decision making, social norms or collective action, while in parallel we have the opportunity to integrate both qualitative and quantitative evidencebased knowledge in a virtual social laboratory.

\section{Conclusions}

The present study showed that $\mathrm{ABM}$ can be useful to understand the inherent complexity of social inequalities in health. These initial findings suggest that the use of ABM may enhance our understanding of interactive mechanisms between social and environmental determinants of health. 
The model suggested that, although poor health outcomes may directly occur through the action of individual determinants, social inequalities generally emerge and reproduce by non-linear associations and complex multivariate data structures.

Author Contributions: J.A.-G. contributed to the conceptualization and the development of the simulation models; V.S.-L. implemented the neural network models and described the results; both authors contributed to the writing of the document.

Funding: This research received no external funding.

Conflicts of Interest: The authors declare no conflict of interest.

\section{References}

1. Rutter, H.; Savona, N.; Glonti, K.; Bibby, J.; Cummins, S.; Finegood, D.T.; Greaves, F.; Harper, L.; Hawe, P.; Moore, L.; et al. The need for a complex systems model of evidence for public health. Lancet 2017, 390, 26022604.

2. Wilkinson, R.G.; Marmot, M. (Eds.) Social Determinants of Health: The Solid Facts; WHO Regional Office for Europe: Copenhagen, Denmark, 2003.

3. Alvarez-Galvez, J.; Suarez-Lledo, V.; Martinez-Cousinou, G.; Muniategui-Azkona, E.; Gonzalez-Portillo, A. The impact of financial crisis and austerity policies in Andalusia, Spain: Disentangling the mechanisms of social inequalities in health through the perceptions and experiences of experts and the general population. Int. J. Equity Health 2019, 18, 108.

4. Alvarez-Galvez, J.; Jaime-Castillo, A.M. The impact of social expenditure on health inequalities in Europe. Soc. Sci. Med. 2018, 200, 9-18.

5. MacKenbach, J.P. The persistence of health inequalities in modern welfare states: The explanation of a paradox. Soc. Sci. Med. 2012, 75, 761-769.

6. Speybroeck, N.; Van Malderen, C.; Harper, S.; Müller, B.; Devleesschauwer, B. Simulation Models for Socioeconomic Inequalities in Health: A Systematic Review. Int. J. Environ. Res. Public Health 2013, 10, 57505780.

7. Alvarez-Galvez, J. Discovering complex interrelationships between socioeconomic status and health in Europe: A case study applying Bayesian Networks. Soc. Sci. Res. 2016, 56, 133-143.

8. Galea, S.; Riddle, M.; Kaplan, G.A. Causal thinking and complex system approaches in epidemiology. Int. J. Epidemiol. 2010, 39, 97-106.

9. ESS Round 7: European Social Survey Round 7 Data; Data File Edition 2.2; NSD-Norwegian Centre for Research Data: Bergen, Norway, 2014. doi:10.21338/NSD-ESS7-2014.

10. Yang, Y.; Roux, A.V.D.; Auchincloss, A.H.; Rodriguez, D.; Brown, D. Exploring walking differences by socioeconomic status using a spatial agent-based model. Health Place 2012, 18, 96-99.

11. Alvarez-Galvez, J. Computational Simulation Methods. In The International Encyclopedia of Communication Research Methods; Wiley: Hoboken, NJ, USA, 2017; Volume 1-11.

12. Diaz, R.; Behr, J.G.; Tulpule, M. A System Dynamics Model for Simulating Ambulatory Health Care Demands. Simul. Healthc. J. Soc. Simul. Healthc. 2012, 7, 243-250.

13. Auchincloss, A.H.; Riolo, R.L.; Brown, D.; Cook, J.; Roux, A.V.D. An Agent-Based Model of Income Inequalities in Diet in the Context of Residential Segregation. Am. J. Prev. Med. 2011, 40, 303-311.

14. Alvarez-Galvez, J. Network models of minority opinion spreading: Using agent-based modeling to study possible scenarios of social contagion. Soc. Sci. Comput. Rev. 2016, 34, 567-581.

15. Mahamoud, A.; Roche, B.; Homer, J. Modelling the social determinants of health and simulating short-term and long-term intervention impacts for the city of Toronto, Canada. Soc. Sci. Med. 2013, 93, 247-255.

(c) 2019 by the authors. Licensee MDPI, Basel, Switzerland. This article is an open access article distributed under the terms and conditions of the Creative Commons Attribution (CC BY) license (http://creativecommons.org/licenses/by/4.0/). 